\title{
Estudios sobre la desmotivación del alumnado en el aprendizaje formal de lenguas extranjeras: estado de la cuestión
}

\author{
Learning Demotivation Studies in the Foreign Language \\ Contexts: State of the Art
}

María del Carmen Méndez Santos [macms@ua.es]

Universitat d'Alacant, España

\section{RESUMEN}

La desmotivación es un fenómeno ampliamente presente en las aulas de lenguas extranjeras como queda constatado en la revisión bibliográfica que se presenta a continuación. Además de resumir y de presentar los resultados de las investigaciones llevadas a cabo en múltiples países, también se acota y define la terminología de trabajo y se traza la evolución histórica del constructo teórico. Asimismo, se describe la metodología empleada y se establecen líneas de trabajo futuras sobre el campo de la desmotivación en el aprendizaje de lenguas extranjeras en contextos formales.

\section{Palabras Clave}

Amotivación; desmotivación; motivación; metodología; revisión de literatura científica

\begin{abstract}
It is widely accepted that demotivation is a phenomenon present in the foreign language classrooms, as it will be shown in the bibliographic review presented below. In addition to summarizing and presenting the results of the research carried out in multiple countries, the terminology is also defined, and the historical evolution of the theoretical construct is traced. Likewise, the methodology that is used here is described and future lines of work are drawn on the field of demotivation in the learning of foreign languages in formal contexts.
\end{abstract}

\section{KEYWORDS}

Amotivation; demotivation; motivation; method; state of the art

RECIBIDO 2019-02-5; ACEPTADO 2019-03-30

El presente artículo forma parte de un proyecto de investigación en lingüística aplicada sobre la desmotivación en el aprendizaje de español como lengua extranjera financiado a través de una beca de movilidad para jóvenes investigadores José Castillejo del Ministerio de Educación y de Formación Profesional (CAS2018/00005) 


\section{Introducción}

Las políticas lingüísticas del siglo XXI de los gobiernos han tomado la enseñanza de lenguas extranjeras (en adelante, LE) como una estrategia fundamental para, entre otros objetivos, fomentar la comunicación intercultural, favorecer los intercambios económicos y la movilidad de trabajadores, y promover la integración de personas migrantes (Consejo de la Unión Europea 2008; Gottlieb 2011; Hu 2007; Department of Education and Skills 2017; Department of Education of the Republic of Philippines 2012). Esto ha conllevado su incorporación de manera general en los sistemas educativos, aunque no siempre con los resultados deseados. Es necesario revisar los éxitos y los errores pasados porque, a pesar de la voluntad institucional, el grado de fracaso a nivel mundial en el aprendizaje de idiomas es alto (Dörnyei 2001: 146; Dörnyei y Ryan 2015: 100). A nivel macroestructural hay trabajos sobre las razones por las que los programas educativos no han logrado todos los retos propuestos. En esta línea destacan los trabajos de Kaplan, Baldauf y Kamwangamalu (2011) y Baldauf, Kaplan, Kamwangamalu y Bryant (2011). En ellos se establecen doce factores clave que han llevado a muchos sistemas a resentirse en su desarrollo como la falta de horas de exposición a la lengua meta, la falta de formación del profesorado, la inadecuación de los materiales o la carencia de recursos, las discordancias entre las metodologías empleadas y los resultados esperados sobre la competencia de los aprendientes, etc. Algunos de esos factores que hacen fracasar los planes de implantación de la enseñanza de lenguas extranjeras suceden en el nivel de aula y por ello repercuten directamente en las actitudes del alumnado llevándolos incluso a perder el interés y la motivación por el aprendizaje. El análisis del origen de esa desmotivación es, pues, una cuestión fundamental para evaluar y mejorar los programas y planes de enseñanza.

Por ello, en este trabajo queremos presentar un estado de la cuestión de los estudios sobre aquellos factores desmotivadores presentes en el nivel del aula en la instrucción formal de LE. Seguimos así la línea del artículo de Kim y Kim (2013) en el que realizaron una revisión de literatura científica en inglés. La novedad que aporta este trabajo es, en primer lugar, la actualización de datos, pero también la mayor cantidad de estudios consultados y de procedencias más diversas, así como el hecho de que sea el primero de estas características redactado en español.

Para cumplir esos objetivos la estructura de este artículo se ha distribuido en un apartado de contextualización histórica del constructo teórico de la desmotivación, una revisión prolija de la literatura científica y un apartado final de valoración de la situación y de diagnóstico de necesidades.

\section{Evolución histórica del constructo teórico de la motivación y de la desmotivación}

La motivación en la enseñanza de lenguas extranjeras forma parte de una tríada -observando el fenómeno desde una perspectiva muy genérica- de variables individuales que junto con los aspectos cognitivos y afectivos se han aceptado como determinantes a la hora de entender el proceso en sí mismo y sus resultados. Los primeros estudios sobre motivación surgieron en el contexto canadiense de la mano de Gardner y Lambert (1972) en los años 50 (Kikuchi 2015: 20) en una comunidad angloparlante, pero con una fuerte presencia de hablantes de francés. Esto determinó su 
concepción teórica de las razones por las que las personas se sienten motivadas para aprender otra lengua. En ese caso establecieron dos fuerzas motivadoras: el deseo de integración en la cultura porque se tienen sentimientos positivos hacia ella (motivación integradora) y un deseo práctico y utilitario (motivación instrumental). Este modelo fue denominado Socio-educational model of Second Language Acquisition y fue el marco teórico de una etapa muy fructífera de estudios que siguieron esta línea hasta los años 90 (incluso en la actualidad). No obstante, la perspectiva del tiempo ha dejado en evidencia, como señala (Kim 2015: 30), que este modelo teórico no es adecuado en contextos donde la L2 no es estudiada para la comunicación porque haya una comunidad lingüística de hablantes nativos cerca. De hecho, como señala McClelland (2000: 102) para lenguas internacionales como el inglés es posible que el interés por aprenderlo sea más por relacionarse con extranjeros en general, que con hablantes nativos en particular ${ }^{1}$. Esta fase sociopsicológica se vio superada, como señala Kikuchi (2015: 21), por la etapa cognitivo-situacional. En los 90 los investigadores entendieron que era necesario encontrar los puntos de relación entre la motivación por aprender lenguas y la teoría psicológica y educativa. Además, se produjo un cambio de perspectiva al dar más importancia a los contextos específicos de cada situación que a las actitudes hacia las comunidades de la L2 en general. Es durante este tiempo cuando surgen teorías como la de la atribución de Weiner (1992) que sostiene que las razones subjetivas a las que atribuimos el fracaso o éxito anterior condicionan la motivación para iniciar posteriores acciones; la de Atkinson y Raynor (1974) que determinaron que la aparición de la motivación está relacionada con el hecho de que un aprendiente busque el éxito; la teoría de la autodeterminación de Decy y Ryan (1985) que se centra en cómo los individuos actúan respecto al objetivo (motivación intrínseca o extrínseca); la de Bandura (1997) que describe la valoración que tienen las personas sobre su propia eficacia personal y cómo ello determina su motivación, perseverancia y esfuerzo dedicado al aprendizaje o la de Covington (1998: 36) que establece un modelo cuadripolar que además de considerar la esperanza de éxito, añade el factor de la evitación del fracaso que se relaciona con la desmoralización (Covington 1998: 70). Todas estas teorías (y otras de la época) entendían la motivación como un estado cuyo origen se situaba solo en un factor (o un par de ellos) y no se prestaba atención a los cambios que esta experimentaba en su intensidad a lo largo del tiempo. A pesar de que iban apuntando factores fundamentales que condicionan de hecho la motivación y su mantenimiento, no dejaban de ser parciales y de dejar recaer todo el peso sobre solo una razón, con lo que se perdía una visión de conjunto sobre más elementos que estaban en juego. El siglo XX dio paso a una nueva concepción. Se cambió la perspectiva estática (se está o no se está motivado) por una dinámica y se dejó atrás también la perspectiva monofactorial dando lugar así a la etapa denominada "orientada al proceso". De esta época son especialmente relevantes los trabajos de Williams y Burden (1997), Dörnyei y Otto (1998, citado en Dörnyei 2001) y Ushioda (citado en Dörnyei 2001). Dörnyei (1994) estableció diferentes niveles que afectan a la motivación al diferenciar el nivel de la lengua (en la que recoge la tradición sobre la orientación integradora y la instrumental); el nivel del alumnado (donde aúna aspectos de teorías de la etapa anterior como las atribuciones causales, la eficacia personal percibida, la competencia, la ansiedad, la necesidad

1 Es posible que esta afirmación sea aplicable también para el español dada su condición de segunda lengua materna más hablada en el mundo por detrás del mandarín (Instituto Cervantes 2018: 5), por lo que los deseos de los aprendices de asimilarse a la cultura meta no siempre serán un objetivo mayoritario o único, hecho que es conveniente tener claro desde el principio de un curso para hacer un buen análisis de necesidades. 
de obtener logros...) y el nivel de la situación del aprendizaje donde se citan aspectos relacionados con la propia asignatura (interés, relevancia...); con el profesorado (tipo de autoridad, estilo de enseñanza) y con el grupo (normas, cohesión, estructura). Williams y Burden (1997) establecieron otra clasificación de condicionantes de la motivación para el aprendizaje de LE basada en factores internos (sexo, edad, estados afectivos, actitudes, sentimiento de agencia, valor percibido de las actividades...) y otros externos (profesorado, grupo, entorno, contexto en general). Como se puede deducir, esos análisis más complejos ofrecían unas teorías con mayor potencial explicativo. Además, el abandono de la idea estática dio paso a una concepción de la motivación como un proceso cambiante y maleable introduciendo el eje temporal (fase preaccional, accional y postaccional) (Dörnyei 2001; Dörnyei y Ottó 1998). Posteriormente Dörnyei (2009) desarrolló, siguiendo esta línea, la teoría del sistema de los posibles yoes ( 22 motivational self-system).

La última etapa de la evolución conceptual sobre el constructo de la motivación en el aprendizaje de lenguas extranjeras abarca el período actual denominado sociodinámico. Como apunta Ryan (2013: 494) esta fase tiene influencias de la teoría sociocultural por la que el contexto ya no se concibe como algo externo, sino como algo que interactúa con el individuo. La motivación es pues considerada como una fuerza procesual que necesita iniciarse, mantenerse y evaluarse teniendo en cuenta todos los factores internos, externos y contextuales que interactúan con ella en una situación dada de modo que en esa relación de interdependencia todos los elementos implicados en el proceso de enseñanza-aprendizaje se afectan mutuamente. Al tener en cuenta que la motivación no surge como consecuencia de la presencia o ausencia de manera aislada de una causa, sino que lo hace de una forma interdependiente y que cambia a lo largo del tiempo es necesario emplear otro marco teórico que explique todos estos aspectos. De este modo, la teoría de los sistemas dinámicos complejos ha encontrado su espacio porque, como señalan Dörnyei, MacIntyre y Henry (2015: 1), parece que describe adecuadamente el complejo y desconcertante fenómeno del aprendizaje de lenguas. El reajuste de este marco teórico deja atrás las relaciones causa-efecto simples entre variables aisladas. Además de tener en cuenta la variabilidad temporal, otro de los elementos implicados es esta concepción es el hecho de que se considera cada sistema de manera contextual analizando así, la organización de esos elementos y de sus patrones de comportamiento que surgen en ese contexto dado. Por lo tanto, esta concepción no ofrece moldes predeterminados o fórmulas sobre qué factor es más fundamental para promover la motivación, sino que analiza el sistema que ha emergido e intenta analizar los fenómenos que lo rigen. De hecho, en ese sistema pueden detectarse estados atractores de cambio que pueden modificar el sistema (Hiver 2015: 20) en sí mismo para crear o bien una inercia positiva o negativa. Estos atractores serán más potentes y crearan una inercia más fuerte cuanto mayor presencia tengan en el tiempo y con la intensidad con la que se presenten. Si entendemos la motivación y la desmotivación como parte de esos sistemas dinámicos complejos, la mayor presencia tanto en el tiempo como intensidad de la primera, y la minimización o eliminación de la existencia de la segunda, tendrán como resultado un sistema con un carácter positivo en cuanto a las fuerzas motivacionales. Habiendo reconocido pues la existencia de estados atractores negativos conviene acotar también ese fenómeno.

No fue hasta 1993 cuando la desmotivación suscitó el interés de los investigadores si tomamos como referencia la publicación de la investigación de Chambers en The Language Learning Journal, pero cuyo verdadero esplendor tuvo lugar tras los trabajos de Dörnyei (2001) y Kikuchi 
y Sakai (2009), que se han convertido en una referencia global. No obstante, para analizarlos y entenderlos es necesario acotar y definir la terminología.

En primer lugar, es necesario diferenciar la amotivación (ausencia de motivación absoluta, Vallerand et alii 1992) de la desmotivación. Por lo tanto, una persona desmotivada será aquella que ya estuvo motivada para aprender una LE, pero que ha perdido su compromiso o interés por alguna razón (Dörnyei 2001: 142). Se puede colegir de esto que la motivación está expuesta a cambios a manos de fuerzas positivas y negativas (Dörnyei 2001: 141) que pueden influir en los comportamientos de los estudiantes. Si se experimentan con mucha intensidad fuerzas negativas, como decíamos, esto puede conducir a una fuerte y prolongada aparición de episodios de desmotivación que desemboquen en un rechazo parcial o completo del aprendizaje, de ahí la importancia de su estudio y comprensión.

La desmotivación fue definida en el ámbito de la enseñanza de LE por primera vez -al menos que tengamos constancia- por Dörnyei (2001: 143). Si bien ya había referencias a la idea de la "pérdida de energía" para aprender. En particular, Covington (1998: 70-71) hablaba de desmoralización y de déficit de motivación:

The practical implications of the learner helplessness paradigm have not been listed on researchers. If it is try that the essence of learned helplessness, or what might be called a motivational deficit, is the belief that one is unable to control events for lack of ability, then perhaps teachers can promote a renewed sense of hope by encouraging students to change their explanations for failure from being the result of low ability to being a lack of effort [...] This reasoning has given rise to a body of research referred to collectively as attribution retraining [...] The effectiveness of attributional retraining will likely depend on where on the downward spiral of demoralization students find themselves. (Covington 1998: 70-71)

No obstante, el término aceptado y usado en los estudios, quizás por su transparencia, es desmotivación. Dörnyei (2001: 143) describió esta como "specific external forces that reduces or diminish the motivational basis of a behavioural intention or an ongoing action". Sin embargo, como señala Kikuchi (2015:3), esta definición adolece de dos limitaciones. En primer lugar, no se puede identificar el fenómeno (desmotivación) con los factores que lo producen ("forces", los factores desmotivadores). Esta matización de Kikuchi lleva a que se diferencie conceptualmente entre el proceso (desmotivación) y los factores desencadenantes (desmotivadores) de una acción (desmotivar) que reduce el nivel de motivación de las personas que lo experimentan (desmotivadas). Es más, las personas desmotivadas pueden volver a ser motivadas (remotivación). Asimismo, no se puede circunscribir las fuerzas provocadoras de la desmotivación como un factor completa y exclusivamente externo al individuo ("external" forces), ya que parece que cuestiones como el sexo, el nivel de autoestima o la percepción sobre experiencias pasadas condicionan también la posibilidad de experimentar episodios de desmotivación.

En cuanto a las características del fenómeno de la desmotivación conviene apuntar que esta influye negativamente en el aprendizaje del alumnado (Kikuchi 2015) porque debilita -que no anula necesariamente- la voluntad del aprendiente y disminuye su autoestima (Falout y Falout 2005: 280). Además, del mismo modo que la motivación, esta fluctúa en el tiempo (Falout 2012: 2) y depende de múltiples factores y es maleable (Kaivanpanah y Ghasemi 2011: 91). Los factores 
desmotivadores hacen que el aprendizaje sea menos placentero y en consecuencia que los resultados esperados del aprendizaje sean más difíciles de alcanzar (Kaivanpanah y Ghasemi 2011: 90). Del mismo modo que en los estudios recientes de motivación que se llevan a cabo bajo el amparo de los sistemas dinámicos complejos (Dörnyei, MacIntyre y Henry 2015), el fenómeno de la desmotivación debe ser comprendido de la misma manera. Esta conceptualización del fenómeno es fundamental para entender que la desmotivación puede aparecer como consecuencia de la interacción de los múltiples factores implicados en cualquier momento en la enseñanza reglada y cómo esta puede ser algo momentáneo, esporádico o muy frecuente y experimentarse con diferentes grados de intensidad. En función de en qué momento, cómo y respecto a qué elementos se produzca esta desmotivación se podrán tomar un tipo de medidas u otras en el aula.

Por lo tanto, las posibles implicaciones didácticas para la enseñanza de LE de esta concepción de la fuerza y duración de los posibles estados atractores positivos y negativos es que la reducción a la mínima expresión de estados atractores negativos es un objetivo legítimo. De hecho, Christophel y Gorham (1995: 302) encontraron en su estudio que la fuerza más intensa en la motivación era precisamente la ausencia de factores desmotivadores. Ahora bien, ¿cuáles son los factores que provocan la desmotivación? Las diferentes investigaciones que se han llevado a cabo y que se presentan a continuación dibujan un panorama muy específico de los factores comunes a todos los contextos de instrucción formal y también de aquellos más particulares de cada contexto sociocultural. No obstante, es cierto que dada su época histórica en la mayoría de ellas se sigue un marco teórico ya superado, si bien es necesario conocerlas igualmente para poder sentar las bases de nuevas investigaciones sobre esos mismos contextos teniendo en cuenta todos los factores que ya se hayan determinado o atestiguado con el fin de actualizar y quizás afinar algunas afirmaciones o resultados.

\section{Revisión de literatura científica}

La mayoría de los trabajos documentados sobre desmotivación se centran en la enseñanza del inglés como LE. Sobre su metodología -que explicaremos con detalle para quienes se planteen realizar algún TFG, TFM o tesis doctoral- y resultados daremos parte. No obstante, también se presentan algunos estudios referidos a otras lenguas como el japonés o el español. Todos ellos se disponen separados por contextos geográficos y en orden cronológico para que los docentes o investigadores de cada zona puedan tener más fácil encontrar los antecedentes de sus potenciales estudios. Por último, cabe precisar que los trabajos aquí resumidos son aquellos a los que ha sido posible acceder y revisar con detalle, aunque haya más; sin embargo, creemos que es una representación amplia y profunda de los estudios ya realizados y de las metodologías más empleadas.

Como se señalaba anteriormente el trabajo Chambers (1993) abrió una nueva línea de investigación. Así, este autor formuló 14 preguntas a 191 estudiantes de cuatro escuelas de Reino Unido y constató que algunos estaban desmotivados porque eran forzados a elegir un curso de lengua y otros porque no veían la razón de estudiar otras lenguas al ser la suya una de las más habladas, si bien la mayoría entendía la importancia de estudiar LE. Una de las conclusiones más interesantes de aquel estudio fue que quienes estaban desmotivados poseían una autoestima muy baja. 
Ushioda (1998, en Dörnyei 2001) trabajó con veinte estudiantes irlandeses de francés como LE mediante entrevistas abiertas en las que identificó ocho dimensiones relacionadas con la presencia de la desmotivación: el interés académico, el disfrute de la lengua, el nivel de competencia deseado, sus objetivos personales, tener una historia positiva de aprendizaje, la satisfacción personal, los sentimientos hacia la gente francesa y presiones externas. Según su trabajo, casi sin excepciones, todos los factores desmotivadores están relacionados con el modo de aprender las LE en un contexto formal institucionalizado.

Oxford en 1998 (en Dörnyei 2001) requirió redacciones a 250 estudiantes americanos sobre sus experiencias de aprendizaje durante cinco años y el factor más desmotivador que emergió era el profesorado. En particular se señalaban cuatro facetas: la relación del profesorado con los estudiantes, la actitud del docente hacia el curso o los materiales, un conflicto entre el estilo del docente y los estudiantes y la naturaleza de las actividades de la clase.

La investigación del propio Dörnyei (2001: 151 y ss.) se llevó a cabo haciendo entrevistas estructuradas largas con cincuenta estudiantes de secundaria de inglés y de alemán como LE que ya estaban identificados como desmotivados. Esto arrojó un listado de los siguientes factores desmotivadores que se correspondían con el 75\% de los comentarios realizados: 1 . El docente (40\% de los comentarios) (personalidad, compromiso, competencia, método de enseñanza); 2. Condiciones de la enseñanza inadecuadas (grupos muy grandes, inadecuación del nivel, cambio frecuente de profesorado); 3. Confianza en uno mismo reducida por pasadas experiencias de fracaso o falta de éxito; 4. Actitudes negativas hacia la LE estudiada; 5. Naturaleza obligatoria de la asignatura; 6. Interferencias con otras lenguas que los aprendices están estudiando; 7. Actitudes negativas hacia la comunidad de habla de la LE; 8. Actitudes de los miembros del grupo y 9. Manual de clase.

Estos trabajos, en especial el de Dörnyei, fueron continuados y replicados con especial entusiasmo en países asiáticos, quizás promovidos, por un lado, por las deficiencias de los sistemas educativos $y$, en parte, por la perspectiva sesgada de quien desde fuera identificaba ciertos rasgos presentes en esas aulas como desinterés, apatía, renuencia, desmotivación y por las creencias sobre lo que convenía más al "alumnado asiático". Los trabajos de investigación realizados han demostrado que esas generalizaciones en términos absolutos no son sostenibles (Cheng 2000; Azpiroz 2013; Muñoz Sanz 2015; Mendoza Puertas 2017), dado que, además de las incuestionables cuestiones culturales presentes, hay otros muchos factores contextuales e individuales (sexo o nivel de competencia, por ejemplo) que condicionan la disposición a participar en las aulas de los estudiantes de origen asiático.

Falout y Maruyama (2004), Arai (2004) y Falout y Falout (2005) trabajaron en el contexto japonés. En el caso del primer estudio, Falout y Maruyama (ibídem), siguiendo las líneas marcadas por Dörnyei, diseñaron un cuestionario de 49 ítems, usando escalas de Likert. Una de las conclusiones más importantes a las que llegaron fue la diferencia del grado de desmotivación entre los estudiantes con alto y con bajo nivel de competencia lingüística.

Hasegawa (2004) trabajó con 125 jóvenes estudiantes de instituto (60 hombres y 65 mujeres) de Fukushima y con 98 estudiantes de últimos años de instituto provenientes de Kanagawa. Formuló un cuestionario de 6 preguntas (¿Te gusta estudiar inglés? ¿Cómo de buenas son tus notas en inglés? ¿Qué cosas te gustan en tus clases de inglés? ¿Qué cosas no te gustan de tu clase de inglés? ¿Pierdes a veces tu interés en aprender inglés? y, una sexta, de corte abierto también para quienes habían contestado que habían perdido su interés alguna vez para explicar por qué y cómo) y lo pasó 
durante las horas de clase. En este estudio la escala de tiempo y el paso de la escolarización parecen haber marcado las tendencias porque entre los estudiantes de último año solamente uno decía que le gustaba estudiar inglés mucho, al contrario que con los jóvenes entre los que a un $9.6 \%$ le gustaba mucho y a un 31.2\% les gustaba. En su análisis halló una fuerte correlación entre las notas que obtenían los estudiantes y su grado de motivación. Las categorías desmotivadoras que determinó la investigadora, en este orden, fueron 1) la naturaleza de las actividades en clase (repetitivas, memorísticas...), 2) la actitud del profesorado, 3) actitudes negativas hacia el aprendizaje de inglés, 4) ansiedad ante la exposición pública en clase, 5) hacer exámenes y obtener malos resultados, 6) la atmósfera de clase (poca sintonía con los compañeros).

Muchos otros investigadores han realizado sus trabajos en el contexto nipón como en el caso de Tsuchiya (2004a, 2004b, 2006a, 2006b), Hamada y Kito (2008), Falout, Elwood y Hood (2009), Fujikawa (2013), Burden (2015), Ohata (2018), entre otros. No obstante, si hay un trabajo de referencia que ha tenido un fuerte impacto a nivel global, son los de Kikuchi y Sakai (2009) y Kikuchi (2015).

Kikuchi y Sakai (2009) desarrollaron un cuestionario de 35 ítems que pasaron a 112 estudiantes de inglés de universidades privadas de Tokio y Shizuoka. Este se ha convertido en una referencia como cuestionario validado y se ha traducido a otros muchos idiomas. El resultado en particular de su investigación acotó cinco factores desmotivadores usando el análisis factorial, a saber, a) manuales de clase, b) condiciones de enseñanza inadecuadas; c) resultados de los exámenes, d) métodos no comunicativos y e) competencia del profesorado y estilo de enseñanza.

Kikuchi, ya en solitario, en 2015 publicó un libro llamado Demotivation in Second language Acquisition. Insights from Japan que puede considerarse una de las pocas obras dedicadas en exclusiva -si no la única- a este tema. En él hace una revisión de los estudios anteriores y acota la terminología de trabajo. Además, presenta un estudio sobre la desmotivación y se explica el desarrollo del cuestionario Sakai-Kikuchi. Entre las principales conclusiones de su trabajo es posible destacar que atestiguaron que los aprendices menos motivados eran los más sensibles a la presencia de desmotivadores y que los que estaban relativamente motivados para aprender tenían más disposición a obviar aspectos negativos del ambiente de aprendizaje. En cuanto a la variable de sexo se probó que los hombres eran más sensibles a factores desmotivadores relacionados con el ambiente de aprendizaje y que la falta de apoyo puede hacer que estos se desmotiven más. Además, también describieron que los hombres eran más proclives a desmotivarse en una clase en la que los medios visuales y auditivos estaban ausentes y en la que las explicaciones eran solo verbales. Por último, en este trabajo se revisan estrategias de motivación y se ofrece un capítulo dedicado a líneas de trabajo futuras.

Esta misma línea de trabajos sobre desmotivación fue seguida en países como Corea, Vietnam, Taiwán, China, etc.

En lo que respecta a Corea podemos informar de los siguientes estudios. Kim y Seo (2012) llevaron a cabo un estudio mixto con estudiantes coreanos de escuelas elementales y sus profesores. El cuestionario fue respondido por 6301 estudiantes de entre el tercer y el sexto grado. Los resultados atribuyeron la desmotivación del alumnado a tres elementos: el impacto negativo del profesorado como la discordancia entre las necesidades del alumno y el interés y paciencia del docente, el exceso de expectativas sobre el nivel de competencia y la creciente diferencia de nivel entre alumnos. 
Li y Zhou (2013) pasaron un cuestionario de 40 ítems a estudiantes de China y Corea y realizaron también entrevistas abiertas. Los factores desmotivadores mencionados fueron en el caso de los aprendices chinos: las estrategias de aprendizaje, las actitudes negativas, el profesorado, el ambiente de aprendizaje, condiciones inadecuadas y la falta de confianza en sí mismos. En el caso de Corea se constató la presencia de factores desmotivadores relacionados con la falta de confianza, la presión de los compañeros, condiciones inadecuadas y el entorno de aprendizaje.

Kim (2015) corroboró que las variables que condicionan la presencia de los factores desmotivadores no solo son externas porque en su estudio se constató que los aprendices con mayor competencia eran más sensibles a las fuerzas externas desmotivadoras y los alumnos con un nivel de competencia menor eran más susceptibles a desmotivarse por factores internos como la sensación de incapacidad.

Kim y Kim (2015) analizaron la desmotivación en estudiantes ancianos que participaban de un instituto que provee educación complementaria para sacarse el graduado en Seúl. Un total de 420 estudiantes respondieron un cuestionario de 47 ítems con escalas de Likert. Los resultados indicaron tres constructos desmotivadores: la percepción negativa de la significatividad del aprendizaje, las dificultades aprendiendo inglés y la presión para aprobar un examen final de graduación.

Kim, Kim y Kim (2017) trabajaron con 869 estudiantes universitarios coreanos mediante un cuestionario de cuatro secciones con preguntas en formato de respuesta con escala de Likert. Así, detectaron seis factores desmotivadores en su contexto: la percepción negativa de los países anglófonos, la naturaleza obligatoria del aprendizaje de inglés, manuales o tareas inadecuadas, baja autoestima, ambiente de aprendizaje desalentador y profesorado no solícito. Sin embargo, no solo se limitaron a listarlos, sino que fueron más allá y también describieron siete factores que fomentan la resiliencia ante la adversidad: satisfacción con la vida, sociabilidad, eficacia comunicativa, competencia estratégica, adaptación metacognitiva, optimismo y entereza.

Otros estudios en este contexto nacional han sido realizados por Jung (2011), Zach (2016), Kim y Kim (2016) o Song y Kim (2017). Este último abarca una perspectiva más amplia, ya que trabajaron con dos grupos de estudio uno de estudiantes desmotivados y otro de aquellos que habían manifestado desmotivación seguida de la remotivación. Este último grupo reportó que la remotivación venía tanto de factores externos (los cambios en la metodología de enseñanza y la inspiración de los compañeros de clase), como internos (aumento de la conciencia de la importancia de aprender inglés para el futuro, más experiencias de éxito y mayor interés por la cultura meta).

Trabajos similares han sido realizados en Taiwán de entre los que destacamos los de Shu-Chen (2012) por centrarse en si los exámenes son motivadores o desmotivadores y el de Hu (2011) en que profundiza en la relación entre desmotivación y nivel de competencia. En cuanto al primero es posible reseñar que los resultados más llamativos de su estudio mixto con encuestas y ensayos escritos de 744 estudiantes son que el diseño de exámenes y la puesta de notas de tareas están asociadas con la disposición de hacer esfuerzo por aprender por parte del alumnado. En el caso de $\mathrm{Hu}$ (Ibídem)es especialmente destacable el hecho de que las dificultades de aprendizaje son un predictor de nivel de competencia. En particular se constata que el aprendizaje de vocabulario es uno de los contenidos que presentan fuertes dificultades y provocan mayo desmotivación.

De Vietnam destacaremos el trabajo de Trang y Baldauf (2007) por ser uno de los pioneros. Emplearon ensayos de 100 estudiantes sobre sus experiencias de aprendizaje para determinar las razones de la desmotivación, el grado de influencia de esos diferentes factores y cómo superaron 
los alumnos esas experiencias. Los resultados indicaron que había una presencia significativa de desmotivación en las aulas y que algunos de los factores desmotivadores aparecían más frecuentemente que otros, no obstante, no se pudo establecer una escala de dificultad para sobrellevarlos. De hecho, la conciencia de la importancia del aprendizaje de inglés y la determinación por aprender de los estudiantes fueron los elementos más determinantes para superar la desmotivación.

Por original merece una mención también el estudio de Nguyen y Thi Pham (2017), ya que se centran en los aspectos desmotivadores en las clases de lectura. Se empleó un cuestionario de 38 ítems para recoger datos de 115 estudiantes. Esos ítems estaban clasificados en grupos de factores desmotivadores: retroalimentación negativa por parte del profesorado, estudiantes, materiales de lectura e instrucción. Los resultados indican que los más desmotivadores son el feedback negativo por parte del profesorado y la falta de eficacia percibida por los propios estudiantes de sus habilidades.

De Bangladesh citamos el único estudio del país que hemos podido constatar. Quadir (2017) realizó una investigación sobre la enseñanza de inglés a través de una entrevista estructurada tomando como punto de partida los factores listados por Dörnyei en 2001. Para la recogida de datos se entrevistó uno a uno con 36 estudiantes de tres universidades de dos ciudades (Dhaka y Chittagong). El tiempo de cada entrevista variaba entre 40 minutos y 1 hora y fueron grabadas en audio. Los datos fueron analizados mediante una reducción y categorización en matrices para su posterior análisis y verificación. En el trabajo se aneja el modelo de las preguntas de las entrevistas realizadas. Los siete factores desmotivadores que se describieron fueron el profesorado y las experiencias pasadas, como principales fuerzas negativas, y después, por este orden, se mencionaron los tutores privados, la actitud de los miembros del grupo, el equipamiento de la escuela, los manuales, los estudiantes y la actitud de las familias hacia el aprendizaje del inglés. Es un resultado original del contexto bangladeshí el hecho de que se mencione los tutores privados como elemento desmotivador. Parece ser que es una realidad muy extendida, pero sin resultados probados sobre el aprendizaje.

Otros estudios que se han documentado que siguen la línea de Kikuchi y Sakai (2009) son los de Krishnan y Pathan (2013) y Mahen y Pathan (2017) en Pakistán. Estos últimos querían determinar los factores desmotivadores en los estudiantes universitarios de Quetta. Emplearon una muestra de 150 estudiantes de tres disciplinas. El cuestionario empleado fue el de Sakai y Kikuchi, aunque emplearon también el cuestionario AMTB de Gardner (1985) para detectar la orientación instrumental o integradora de su motivación. En esta ocasión los contenidos del curso y los materiales emergieron como los factores más desmotivadores y la motivación instrumental fue la más claramente reportada en la muestra.

Estudios más recientes han sido realizados en Tailandia como el de Boonchuayrod y Getkham (2018) o en Filipinas. En el caso de la investigación tailandesa se empleó una muestra de 158 estudiantes de inglés. Se empleó el cuestionario Sakai-Kikuchi de 35 ítems con escalas de Likert de 5 puntos en inglés y con traducción al tailandés. Los resultados del análisis factorial ofrecieron 6 factores desmotivadores: el profesorado y su estilo de enseñanza; el fracaso; tecnología inadecuada; enfoques no comunicativos; los materiales y el tamaño de la clase y, por último, el ambiente y los compañeros de clase. En Filipinas, Aquino et alii (2016) entrevistaron al profesorado para conocer sus percepciones sobre la desmotivación del alumnado y qué medidas tomaban ante esa situación. En particular destaca que se requiere del profesorado disminuir la presión y la ansiedad 
a través de estrategias didácticas. En Malasia, Fauzi (2014) realizó su trabajo de fin de máster mixto con cuestionario y entrevistas sobre la desmotivación de los estudiantes preuniversitarios malayos con el inglés como LE.

El trabajo de Xuezheng (2014) en China con 700 estudiantes universitarios que participaron en la muestra a través de cuestionarios constató que los factores que más disminuyen la motivación son el bajo grado de motivación integradora en la comunidad meta, la falta de sintonía con el profesorado, una sensación de fuerte dificultad para el aprendizaje durante el curso y un desinterés en estudiar.

Paralelamente a este desarrollo de los estudios de desmotivación en países asiáticos, también se produjo una eclosión en otras áreas geográficas. En Europa el trabajo de Muhonen (2004) sobre la desmotivación en estudiantes de colegio finlandeses representa una gran fuente de citas en subsiguientes investigaciones. En este caso la muestra estaba compuesta de 91 alumnos que rellenaron una tarea de composición escrita sobre sus pensamientos y las cosas que les hacían estar desmotivados. Se analizaron finalmente 86 porque cinco se devolvieron en blanco. La principal fuente de desmotivación es el profesorado, hecho que confirma los estudios de Chambers, Dörnyei y Oxford, entre otros. Los materiales de enseñanza fueron el segundo factor más desmotivador. En este estudio sí se tenía en cuenta el sexo como variable, pero no se encontraron diferencias significativas en la muestra. Esta línea de estudios en el país la continuaron otros estudiantes como Hirvonen (2010) y Amemori (2012).

En la República Checa destacan algunos trabajos de diplomatura como los de Bednářová (2011) o el de Macasová (2014). En el caso de Bednářová (ibídem) se constató que un 93\% de los estudiantes que habían participado habían perdido su interés inicial por el aprendizaje, certificando así que la desmotivación es un fenómeno con graves consecuencias sobre el éxito de los programas educativos. Los factores más prominentes fueron los externos como la crítica al profesorado y sus competencias. Además, el foco en la gramática y la memorización más que sobre las destrezas orales fue descrito como una fuerza desmotivadora. Macasová (ibídem)apreció diferencias en función del sexo respecto a cuánto desmotiva, ya que parece que los hombres son más exigentes y perciben como insuficiente el apoyo del profesorado.

En Israel, Keblawi (2005) requirió a 294 estudiantes árabes de inglés como LE que escribiesen un texto respondiendo a la pregunta "What makes you frustrated or demotivated when studying English?”. Posteriormente realizó entrevistas de seguimiento a 25 estudiantes y a 10 profesores para comprender más plenamente el fenómeno. A pesar de lo que pudiera esperarse los factores contextuales no fueron el principal causante de desmotivación. El profesorado, tanto su estilo como su personalidad, así como las dificultades de la gramática y el aprendizaje de vocabulario se mostraron como fuertes atractores de la desmotivación.

Los estudios sobre la desmotivación en Irán en el aprendizaje de inglés como lengua extranjera gozan de una fuerte salud, dado que se han documentado numerosos trabajos a lo largo de los años y atendiendo a diferentes factores. El primero que tenemos documentado es el de Kaivanpanah y Ghasemi (2011), docentes en Irán que requirieron a 40 estudiantes de secundaria y de universidad que escribiesen en farsi sobre los factores que les desmotivaban aprendiendo inglés. Sus respuestas fueron analizadas y categorizadas en grupos de factores más frecuentemente desmotivadores. Posteriormente se realizó una entrevista semiestructurada a 10 profesores para contrastar la perspectiva del profesorado sobre esos factores desmotivadores. Finalmente elaboraron 
un cuestionario teniendo en cuenta el trabajo de Kikuchi y Sakai (2009) con un número final de ítems de 66. Contestaron este cuestionario redactado en farsi 100 estudiantes de los que se excluyeron 16 por incompletos. Se llevó a cabo un análisis factorial exploratorio con lo que se primó aquellos factores con mayor validez y se eliminaron los redundantes. La versión final determina cinco categorías de factores desmotivadores: el profesorado; los contenidos, materiales y recursos disponibles; las experiencias de fracaso; la actitud hacia la lengua extranjera y la actitud hacia los hablantes de la lengua meta. El cuestionario final se distribuyó a 327 estudiantes en 20 minutos. En el trabajo se aneja el modelo. En este trabajo los contenidos, los materiales y los recursos fueron los desmotivadores más prominentes. En particular, se constató que la falta de uso de tecnología desmotivaba a los estudiantes. También consideraron el sexo como variable y atestiguaron que las mujeres estaban más desmotivadas que los hombres ante el profesorado y las experiencias previas de fracaso. Por el contrario, los hombres se sentían más desmotivados por las actitudes que tienen hacia la comunidad de los hablantes de la lengua meta. Otro trabajo de referencia de ese mismo año fue el de Sharififar y Akbarzadeh (2011) y también emplearon el cuestionario Sakai-Kikuchi con 53 estudiantes universitarios. Al año siguiente de estos estudios pioneros vieron la luz muchos otros sobre la desmotivación en el aprendizaje de inglés en Irán como los de Meshkat y Hassani (2012); Tabatabaei y Molavi (2012), Rastegar, Akbarzadeh y Heidari (2012); Heidari y Riahipour (2012); Ghadirzadeh, Pourabolfathe, Hashtroudi y Shokri (2012). Ghadirzadeh, Pourabolfathe Hashtroudi y Shokri (2012) trabajaron con el modelo persa del cuestionario Sakai-Kikuchi sin más añadidos o adaptaciones. Meshkat y Hassani (ibídem) también emplearon ese cuestionario de referencia, pero añadieron una pregunta más de tipo abierto para completar la información con aquellos factores que no hubiesen sido mencionados. En este estudio con 421 estudiantes de secundaria el factor más prominente fueron las condiciones inadecuadas. La originalidad del trabajo de Tabatabaei y Molavi (ibídem) reside en que emplearon como referencia el cuestionario de pasividad Warrington (2005) y Warrington y Jeffrey (2005), que no ha sido especialmente empleado en la literatura sobre desmotivación. En los anejos se ofrece el modelo adaptado que se proporcionó durante el estudio. Rastegar, Akbarzadeh y Heidari (ibídem) relacionaron la desmotivación con el factor de la ansiedad aplicando la escala de ansiedad en las clases de lenguas extranjeras (FLCAS) y el cuestionario Sakai-Kikuchi, hecho que lo hace muy original en comparación con la mayoría de los que se han llevado a cabo. Los resultados son claros: cuanto más desmotivados están, más alto puntúan en el test de ansiedad y también en el FLCA. En este caso la variable de sexo no obtuvo resultados significativos. El paradigma de estudios iraníes se completó en años posteriores con estudios sobre la desmotivación en la secundaria (Hosseini y Jafari 2014; Rajavi y Hosseini Pozveh 2016); la descripción del constructo de la desmotivación y los desmotivadores (Afrough, Rahimi y Zarafshan 2016; Ghonsooly, Hassanzadeh, Samavarchi y Hamedi 2017) y otras investigaciones más originales que tienen en cuenta otras variables. Llaman particularmente la atención los trabajos de Jahedizadeh, Ghanizadeh y Ghonsooly (2016) y de Arefinezhad y Golaghaei (2014). El primero porque se analiza la corrección de la percepción de las clases, la desmotivación y los objetivos del alumnado para predecir su éxito en el aprendizaje y su posible estado de burnout. Para ello emplearon los trabajos de desmotivación de Kikuchi y Sakai (2009); la versión farsi de la escala Maslach Burnout Inventory Student-Survey (Schaufeli et alii, 2002) y del Achievement Goal Orientation Inventory de Midgley et alii (1998), así como la escala de Gentry y Gable (2001) sobre la percepción de las actividades de clase por parte de los estudiantes. Los re- 
sultados reflejan que 5 de los 6 factores desmotivadores tienen consecuencias significativas sobre el quemado de los estudiantes (materiales de clase, falta de interés, experiencias de fracaso, ambiente de clase y profesorado). Asimismo, se ha mostrado una relación negativa entre el síndrome del alumnado quemado y las aspiraciones de competencia del alumnado. En otras palabras, si el alumnado experimenta el síndrome de estar quemado evitará tener una actitud de búsqueda del dominio de la LE. Por otro lado, los estudiantes que muestran síntomas de estar quemados están condicionados en sus percepciones de las actividades de clase, por ejemplo, disfrute, interés... es decir, si están quemados ya no perciben las actividades como interesantes, divertidas, etc. El trabajo de Arefinezhad y Golaghaei (2014) es original porque se centra en el vocabulario como foco de trabajo, de modo que se analizan los posibles factores desmotivadores en su aprendizaje. A saber, en este estudio se constatan como mayores desmotivadores a la hora de aprender léxico la falta de recursos, el método y los materiales empleados. El sexo es una variable que tener en cuenta ante el aprendizaje de léxico porque para las mujeres se constató que lo más desmotivador era la competencia del profesorado mientras que para los hombres refirieron la metodología, los materiales, el ambiente de clase, las características de las clases, la oportunidad de usar la lengua, las actitudes antes el inglés y el uso de tecnología como potenciales desmotivadores.

En Arabia Saudí como en otros contextos internacionales el inglés es considerado importante por razones económicas, sociales y científicas por lo que el gobierno ha invertido grandes sumas de dinero para promover su aprendizaje. En este contexto, Al-Khairy (2013) realizó un cuestionario de 23 ítems con escalas de Likert diseñado por el mismo, ya que, según se explica, ninguno de los revisados se adaptaba a la realidad saudí y lo distribuyó aleatoriamente a estudiantes del departamento de lenguas extranjeras de la Taif University (150) y del centro de idiomas de la misma universidad (150). El total de respuestas que se procesó fue de 292 usando SPSS y un test T. Al trabajo se aneja el modelo de cuestionario empleado. El factor más desmotivador está relacionado con el profesorado y su personalidad, competencia, falta de interés, etc. Según indica el alumnado prefiere unas clases más activas en la que se usen medios audiovisuales. El segundo factor citado como desmotivador es el tener un gran número de alumnos por clase. En tercer lugar, se constata una desmotivación originada en el discurso del profesorado, en particular, relacionado con la velocidad. En 2015, Gulnaz, Ahmad y Mandouh, realizaron un estudio con estudiantes de varios países del Golfo Pérsico, incluyendo estudiantes saudíes. Además de entrevistar a siete profesores, pasaron a 200 estudiantes de inglés de cuatro universidades, 50 de cada una, un cuestionario de 17 ítems para ser valorados con escalas de Likert de 5 puntos. Las preguntas estaban centradas en núcleos temáticos: el estilo del profesorado; la metodología y técnicas de aula; la falta de determinación del alumnado y sus objetivos para aprender inglés y aspectos personales, sociales y religiosos relacionados con el estudiante. Al-Khasawneh (2017) aplicó, esta vez sí, el cuestionario Sakai-Kikuchi con los 35 ítems con una escala de Likert de 5 puntos. La muestra se realizó con 101 estudiantes de tres facultades de la Universidad Rey Khalid. El análisis se llevó a cabo con un test T. En este caso los resultados muestran que el factor más desmotivador era las características de las clases en particular en aspectos relacionados con clases demasiado saturadas y un papel pasivo por parte del alumnado. En 2017 se defendió una tesis doctoral realizada por Fatemah Hammad Eid Albalawi en la Universidad de Nottingham sobre las actitudes ante el aprendizaje de inglés como lengua extranjera y su relación con la desmotivación. 
El único estudio que ha sido documentado en portugués fue realizado en Brasil (Parente y Pereira da Silva Schneider 2013). Ambas investigadoras buscaban determinar las causas de la motivación y de la desmotivación en el alumnado de $9^{\circ}$ (enseñanza obligatoria, entre 14 y 16 años) al estudiar inglés en una escuela municipal en la ciudad de Palmas. Visitaron y observaron varias sesiones de clase de un grupo de 25 estudiantes de las que se ofrece una descripción. Además, al final de su estancia aplicaron un cuestionario de ocho preguntas teniendo como marco de referencia el constructo de motivación intrínseca y extrínseca. No se ofrece un modelo del cuestionario con lo que no es posible saber si fueron preguntas totalmente abiertas o si se ofrecieron opciones de selección múltiple. Tampoco se explica cómo se codificaron las respuestas en el caso de que estas hubieran sido abiertas. Las preguntas relativas a la motivación y a la desmotivación en particular fueron formuladas de manera parafrástica: $O$ que te dá mais prazer em aprender nas aulas de inglês? y O que te dá menos prazer em aprender nas aulas de inglês? Los resultados a estas dos cuestiones fueron que el $76 \%$ contestó que lo que más le motivaba era la conversación. Los porcentajes de mención a la gramática (14\%), comprensión lectora (4.7\%) y expresión escrita (4.7\%) eran menores con una gran diferencia porcentual. Entre los factores que más desmotivan se menciona la expresión escrita (28.5\%), la lectura (28.5\%), la gramática (19\%) y la conversación (9.5\%). Hay un porcentaje residual de un $5 \%$ que no se especifica a qué corresponde. En la sexta pregunta sobre qué medidas se pueden tomar para hacer las clases más motivadoras (Em sua opinião o que um professor de língua inglesa poderia fazer para tornar as aulas mais motivadoras?) los aprendices señalaron promover dinámicas de grupo (38\%) y usar recursos multimedia (33.3\%) como principales medidas que tomar.

En Marruecos, Baba Khouya (2017) realizó una investigación para comprender los problemas de la enseñanza del inglés en el país en la que participaron 210 estudiantes de segundo curso de bachillerato de seis escuelas secundarias de los que 117 eran mujeres y 84 hombres. Para ello empleó una metodología mixta: cuestionario y ensayo escrito. Los datos cuantitativos fueron analizados teniendo en cuenta la frecuencia y porcentajes, test chi cuadrado y un test $\mathrm{T}$. El cuestionario estaba compuesto de 23 ítems que abarcaban 4 secciones: la primera sección incluía cinco ítems sobre la percepción de los estudiantes del aprendizaje de inglés; la segunda sección con siete ítems versaba sobre el inglés y la desmotivación; la tercera sección, compuesta de seis ítems, se centraba en la relación del profesorado con la desmotivación; la cuarta y última parte estaba compuesta de cinco ítems sobre el ambiente de aprendizaje y la desmotivación. El cuestionario, que no se adjunta al trabajo, fue administrado en árabe dado que el nivel de inglés de los discentes podía afectar a su comprensión y, por ende, a los resultados del estudio. Después de haber rellenado el cuestionario, los doscientos diez estudiantes escribieron un párrafo, también en árabe, en el que debían referir su experiencia: According to your own experience during learning the English language, what are the factors that demotivated you in learning this language? Como justifica el autor se eligió un ensayo para completar el cuestionario que se distribuyó porque este tenía mayoritariamente preguntas cerradas y porque se quería recuperar una información más detallada. Se desechó la idea de realizar entrevistas porque estas demandan una inmediatez interaccional que quizás dificultaría el recuerdo de las experiencias por parte de los estudiantes por lo que la muestra se vería empobrecida. Los seis hallazgos principales del trabajo son que los estudiantes (1) se perciben positivamente como aprendices de inglés, ya que un $80.2 \%$ afirmó considerarse como buen o muy buen estudiante; (2) no mostraron una actitud negativa antes el inglés como 
lengua (un 74.6\% lo negó); (3) valoran positivamente a su profesorado (64.2\% consideraba que eran competentes o muy competentes); (4) apuntan que las clases con muchos estudiantes es el principal factor desmotivador seguido de sus compañeros de clase, el manual y el ambiente de clase; (5) consideran que el ambiente de aprendizaje es muy desmotivador por la falta de recursos y equipamiento y, una última conclusión fue que (6) el sexo no era una variable significativa en los factores desmotivadores. Estos resultados le llevan a proponer una serie de medidas como implicaciones prácticas de este trabajo.

En Turquía se han llevado a cabo diferentes estudios como los de Erdogan y Tunaz (2012) sobre la desmotivación de estudiantes de primaria y secundaria. Para la recolección de datos se empleó un cuestionario de 18 preguntas para responder con escalas de Likert y preguntas abiertas. Estas preguntas estaban agrupadas en tres grupos de contenido: motivaciones del alumnado, motivación relacionada con el profesorado y motivación relacionada con el currículo, a razón de seis cuestiones por cada contenido. Participaron 290 aprendices que, además, fueron observados y de los que algunos fueron elegidos aleatoriamente para realizar entrevistas. Aunque no se ofrecen unas conclusiones descriptivas exhaustivas, el cambio de actitudes negativas del profesorado por otras más positivas es la principal implicación didáctica que se propone. El testigo investigador en el país fue recogido por Akay (2017) y su estudio sobre la remotivación y por Çankaya (2018) que ha estudiado los factores desmotivadores en estudiantes universitarios de inglés en Turquía. Akay (ibídem) realizó un estudio exploratorio con 579 estudiantes de instituto. La investigación reveló que el grado de desmotivación era bastante alto y que la remotivación pasaba por tener clases entretenidas, usar recursos tecnológicos y más actividades de expresión oral. Çankaya (ibídem) para la recolección de datos empleó el cuestionario de Sakai-Kikuchi traducido al turco que fue completado por 60 estudiantes de las carreras de turismo, tecnología de los alimentos y marketing. Se completó el cuestionario con dos preguntas abiertas: How motivated are you to learn English? y What are the factors causing motivation and demotivation in English classes? Los factores desmotivadores atestiguados fueron, por este orden, las características de las clases, el ambiente de clase, las experiencias previas de fracaso, la falta de motivación intrínseca, los materiales de clase y el profesorado. Los materiales inadecuados no fueron un factor atestiguado.

Es especialmente reseñable el trabajo de Amador (2018) en su tesis doctoral, ya que introduce un nuevo perfil en el que la importancia de la motivación y de la desmotivación es, si cabe, todavía más crucial: personas migrantes. En este caso usó una entrevista semiestructurada con 8 jóvenes dominicanos que estudiaban la secundaria en Puerto Rico. Entre sus principales hallazgos cabe reseñar que los estudiantes se sentían desmotivados si solo se les hablaba en inglés, si el profesorado desconocía la cultura dominicana, si el docente u otros estudiantes manifestaban comportamientos irrespetuosos (como reírse de los errores o discriminarlos verbalmente) y si compartían clase de EFL con gente nativa de inglés en algún momento.

Como se anunciaba al principio de este artículo, la mayoría de los trabajos documentados sobre desmotivación se centran en la enseñanza del inglés como LE. No obstante, hay algunos estudios referentes a otros idiomas como el de Akita (2017) que reflexiona sobre el aprendizaje de japonés con estudiantes universitarios en Brunei o el de Hamada y Grafström (2014) sobre aprendices de japonés de origen australiano. Asimismo, Aladdin (2013) analizó los factores desmotivadores del aprendizaje de árabe por parte de malayos no musulmanes. En este caso llevó a cabo un cuestionario de preguntas abiertas a 207 estudiantes y 20 entrevistas semiestructuradas. Los resultados 
ofrecieron 9 factores desmotivadores: aquellos relacionados con la materia; el profesorado; las clases; las actitudes hacia la LE; los materiales y los contenidos; la naturaleza obligatoria de la asignatura; las limitaciones temporales; la falta de oportunidades comunicativas en árabe y la falta de capacidad.

En cuanto al español como LE es posible constatar que hay estudios generales sobre motivación que mencionan puntualmente aspectos relativos a la desmotivación. Así, Marins de Andrade y Guijarro Ojeda (2010) realizaron un cuestionario de preguntas abiertas y de respuestas de selección múltiple a 17 estudiantes brasileños de un centro de lenguas público cuyo nivel era intermedio y que llevaban tres años de media estudiando español. Si bien su estudio versaba sobre otros aspectos generales sí formularon una pregunta (7. ¿Qué es lo que más te desmotiva para aprender español?) sobre la desmotivación y los aspectos que se listaron fueron el horario de las clases (nocturno), la gramática de la lengua (considerada difícil) y la falta de tiempo para estudiar. Asimismo, el propio Marins de Andrade (2010) trabajó también sobre las estrategias de remotivación en un trabajo más general con 14 aprendices brasileños sobre estrategias motivacionales (5. Cuando te sientes desmotivado, ¿qué haces para automotivarte, qué estrategias utilizas?). En este caso optó por emplear cuestionarios, entrevistas y observación de clases. Entre las estrategias de automotivación más comunes se reportan hacer lecturas, escuchar música y ver películas en español. Huneault (2009) en otro estudio sobre estrategias motivacionales en el aula de ELE con estudiantes universitarios de Quebec menciona brevemente que la mayoría de los estudiantes desmotivados del estudio atribuyen su abandono a tener que invertir demasiados esfuerzos o a que están insatisfechos con el curso, el programa o el manual empleado.

No obstante, solo ha sido posible encontrar un estudio únicamente centrado en la desmotivación en el aprendizaje de ELE. Bunyamethi (2018) analiza los factores desmotivadores al consultar a 52 alumnos tailandeses de las universidades de Chulalongkorn, Ramkhamhaeng, Thammasat y Khonkean. Siguió el modelo de Kikuchi y Sakai (2009) adaptado al pasar el cuestionario de modo digital. Este se pautó en cuatro partes: una primera de datos de perfil, la segunda donde determinan si se han sentido desmotivados alguna vez o no y, en caso afirmativo, pasaban a la tercera sección basada en escalas de Likert. La última parte era una descripción de una situación en la que se sienten desmotivados. En este trabajo se constata que el $61.53 \%$ de los aprendices se han sentido desmotivados alguna vez. El factor desmotivador más destacado es la falta de interés (34.8\%), seguido de la dificultad lingüística, la falta de salidas profesionales, los contenidos de la clase, experiencias anteriores de fracaso, el profesorado y un porcentaje más residual que menciona el ambiente de clase. La autora señala como particularmente reseñable el hecho de haber constatado las variedades del español y la compatibilización de trabajo y estudios como factores desmotivadores en el estudio, ya que en trabajos anteriores estos no habían sido reportados. Como anejo al trabajo se ofrece un listado de los comentarios que los alumnos esgrimen como desmotivadores.

\section{Conclusiones}

La desmotivación en el aprendizaje de lenguas extranjeras en contextos de enseñanza formal es una realidad innegable como ha quedado patente a la luz de todos los estudios analizados. Dado que su presencia está sujetamente altamente a factores contextuales muy ceñidos al aquí y al aho- 
ra de cada entorno, de cada centro e incluso de cada aula, son necesarios más estudios en cada ocasión que se detecte un debilitamiento de la motivación del alumnado con el fin de acotar el fenómeno y de poder trazar medidas de control y de remotivación.

La mayoría de los estudios se han realizado sobre el inglés como lengua extranjera y de ahí la necesidad de realizar más sobre otros contextos y la enseñanza de otros idiomas, ya que de la propia diversidad lingüística también emanan factores desmotivadores como, por ejemplo, las variedades lingüísticas enseñadas.

La mayoría de los trabajos realizados hasta la actualidad -y en coherencia con sus coordenadas temporales- tenían como referente teórico un constructo sobre la motivación y de la desmotivación, por ende, del período cognitivo-situacional o orientado al proceso. No obstante, dado que los trabajos actuales sobre motivación como los más recientes publicados (Dörnyei, MacIntyre y Henry, 2015) siguen la línea de los sistemas dinámicos complejos como una herramienta más informativa y explicativa, convendría realizar el mismo giro teórico en los estudios de desmotivación. De hecho, en esa perspectiva se sitúan los trabajos de Méndez Santos (2018a, 2018b y en preparación) presentados en diferentes congresos especializados en la enseñanza de lenguas extranjeras.

Los trabajos analizados emplean una metodología cuantitativa y cualitativa, aunque podríamos decir que en la mayoría se usa una modalidad mixta. Los estudios cualitativos han sido más usados para explorar el fenómeno de un modo general y para acotar los factores desmotivadores como categorías y los cualitativos pretendían llegar a conclusiones más descriptivas. Los instrumentos más empleados han sido las entrevistas estructuradas o semiestructuradas; los ensayos o redacciones y los cuestionarios con escalas de Likert de 5 o 6 puntos. Como referencia para elaborar las preguntas o los diferentes cuestionarios empleados se ha seguido, por norma general, el modelo de 9 factores documentado por Dörnyei (2001), a saber los docentes (personalidad, compromiso, competencia, método de enseñanza); las condiciones de la enseñanza inadecuadas (grupos muy grandes, inadecuación del nivel, cambio frecuente de profesorado); la confianza en uno mismo reducida por pasadas experiencias de fracaso o falta de éxito; las actitudes negativas hacia la LE estudiada; la naturaleza obligatoria de la asignatura; las interferencias con otras lenguas que los aprendices están estudiando; las actitudes negativas hacia la comunidad de habla de la LE; las actitudes de los miembros del grupo y los manuales de clase. También se han empleado estas categorías como modelo confirmatorio de los hallazgos en trabajos de carácter más abierto. No obstante, si hay un instrumento especialmente seguido en las investigaciones ha sido el cuestionario Sakai-Kikuchi de 35 ítems que ha sido traducido a varios idiomas. Si bien es cierto que en algunas ocasiones se ha incluido de modo extra alguna pregunta abierta para recoger cualquier otro dato relevante a mayores. Sakai y Kikuchi determinaron 5 factores en su contexto, en lugar de los 9 de Dörnyei, que eran los manuales de clase, las condiciones de enseñanza inadecuadas, los resultados de los exámenes, los métodos no comunicativos y la competencia y el estilo del profesorado. Las respuestas abiertas han sido estudiadas mayoritariamente con un análisis de matrices y trazando clústeres y las cuantitativas con análisis factorial y programas como SPSS. En el caso de nuevas investigaciones sería conveniente recomendar que estas se lleven a cabo con una metodología mixta que aporte la necesaria perspectiva global de la parte cuantitativa y la prolijidad descriptiva que ofrecen los instrumentos cualitativos. 
Asimismo, en lo que respecta a posibles investigaciones futuras es reseñable el hecho de que más variables deberían ser tenidas en cuenta habida cuenta de que se ha demostrado que no solo factores externos condicionan la experimentación de la desmotivación, sino que también está relacionado con aspectos internos y emplear una perspectiva teórica más informativa como la de los sistemas dinámicos complejos. En los trabajos revisados se ha constatado que el grado de desmotivación está condicionado por el nivel de competencia (Falout y Maruyama); el nivel de autoestima (Chambers 1993; Falout y Falout 2005: 280); el sexo (Kaivanpanah y Ghasemi 2011; Kikuchi 2015; Macasová 2014); la edad (Kim y Kim 2015); por los años de escolarización y las notas (Hasegawa 2004); los recursos del aula (Kaivanpanah y Ghasemi 2011); los contenidos y las destrezas (Hu 2011; Nguyen y Thi Pham 2017), entre otras.

Por último, cabe constatar que el siguiente paso después de haber bosquejado trabajos sobre la desmotivación es lógico intentar entender qué estrategias emplean los alumnos que presentan mayor resistencia a la desmotivación (Kim, Kim y Kim 2017) y también detallar qué estrategias remotivadoras pueden llevarse a cabo para mejorar la situación de desmotivación que se haya registrado (Song y Kim 2017; Falout 2012). Todo ello con el fin claro de mejorar el proceso de enseñanza de lenguas extranjeras y de sus resultados, así como de reducir en la medida de lo posible el fracaso escolar.

\section{Referencias bibliográficas}

Afrough, T.; Rahimi, M.; \& Zarafshan, M. (2014). Foreign Language Learning Demotivation: A Construct Validation Study. Procedia - Social and Behavioral Sciences 136, 49-53. <https://bit.ly/2zOXGod>

Akay, C. (2017). Turkish High School Students' English Demotivation and Their Seeking for Remotivation: A Mixed Method Research. Canadian Center of Science and Education, 10 (8), 107-122. < doi: 10.5539/elt. v10n8p107>

Akita, Y. (2017). Motivational and perception changes in learning Japanese in Brunei Darussalam. Border crossing, 7 (2), 249-262. <https://bit.ly/2E8SUVJ>

Aladdin, A. (2013). Demotivating factors in the Arabic language classroom: What demotivates non-Muslim Malaysian learners when it comes to learning Arabic?. Procedia - Social and Behavioral Sciences, 93, 1652-1657. <doi: 10.1016/j.sbspro.2013.10.096>

Albalawi, F.H. (2017). L2 Demotivation among Saudi Learners of English: The Role of the Language Learning Mindsets. Tesis doctoral, University of Nottingham. <https://bit.ly/2SCoFum>

Al-Khairy, M. (2013). English as a foreign language learning demotivational factors as perceived by Saudi undergraduates. European Scientific Journal (ESJ), 9, 32, 365-382. <http://dx.doi.org/10.19044/esj.2013. v9n32p\%25p>

Al-Khasawneh, F. M. (2017). Demotivating Factors Affecting EFL Learning of Saudi Undergraduate Students. International Journal of Language Education and Applied Linguistics (IJLEAL), 6, 25-34. $<$ https://bit.ly/2BXJGtY>

Amador, L. (2018). Motivators and Demotivators of Dominican Immigrant High School ESL Students in Puerto Rico. Tesis doctoral, Walden University, Estados Unidos. $<$ https://bit.ly/2rAbPBr $>$ 
Amamori, M. (2012). Demotivation to learn English among university students in Finland. Trabajo de fin de máster, University of Jyväskylä, Finlandia. <https://bit.ly/2L2JIDD>

Aquino, A. C. et alii (2016). Demotivating factors in learning the English language. Trabajo presentado en DLSU Research Congress, De La Salle University, Manila, Filipinas, 7-9 de marzo de 2016. <https://bit. ly/2UmFgUu>

Arai, K. (2004). What 'demotivates' language learners? Qualitative study on demotivational factors and learners' reactions. Toyo Gakuen Daigaku Kiyo, 12, 39-47.

Arefinezhad, H.; \& Golaghaei, N. (2014). Investigating EFL Learners' attitudes towards demotivating factors in vocabulary learning: a mixed method study. International Journal of Language Learning and Applied Linguistics World, 7 (1), 179-197. <https://bit.ly/2zQll7K>

Atkinson, J.; \& Raynor, J. (Eds.) (1974). Motivation and achievement. Washington: Winston \& Sons.

Azpiroz, M. C. (2013). El estudiante chino de español como lengua extranjera (ELE). Diálogo entre la cultura china y el contexto educativo. Cuadernos de Investigación Educativa, 4, 19, 39-52.

Baba Khouya, Y. (2018). Students Demotivating Factors in the EFL classroom: The Case of Morocco. Advances in Language and Literary Studies, 9, 2, 150-159. <https://bit.ly/2zNi5Km>

Baldauf, R.; Kaplan, R.B.; Kamwangamalu, N.; \& Bryant, P. (2011). Success or failure of primary/second foreign language programmes in Asia: what do the data tell us? Current issues in Language Planning, 12 (2), 309-323.

Bandura, A. (1997). Self-efficacy: The exercise of control. Nueva York: Freeman.

Bednářová, N. (2011). Demotivating Influences for Learning English among Students on Lower Stages of 8-year Grammar School. Trabajo de fin de grado, Universidad de Masaryk, Brno, República Checa. <https://bit. ly/2SyGElb>

Boonchuayrod, P.; \& Getkham, K. (2018). Why Were English Major Students Demotivated in English Language Learning? International Journal of Management and Applied Science, 4, (3), 51-55. <https://bit. ly/2ElEOkx>

Bunyamethi, J. (2018). Los factores desmotivadores en los alumnos tailandeses para el aprendizaje de español como lengua extranjera. Monográfico de SinoELE, 17, 188-202. <https://bit.ly/2SyDEFq >

Burden, P. (2015). Demotivation, amotivation or overmotivation? An Action research project investigating antecedent attitudes to English among Japanese university students. Journal of Okayama Shoka University, $51,1,81-116$.

Çankaya, P. (2018). Demotivation Factors in Foreign Language Learning. Journal of Foreign Language Education and Technology, 3(1), 1-17. <https://bit.ly/2PmsvFT>

Chambers, G. (1993). Taking the 'de' out of demotivation. The Language Learning Journal, 7 (1), 13-16.

Cheng, X. (2000). Asian students' reticence revisited. System, 28, 435-446.

Consejo de la Unión Europea (2008). Resolución del Consejo de 21 de noviembre de 2008 relativa a una estrategia europea en favor del multilingüismo. <https://bit.ly/2QIbgn9>

Covington, M. V. (1998). The will to learn. A guide for motivating Young People. Cambridge: Cambridge University Press.

Christophel, D.; \& Gorham, J. (1995). A test-retest analysis of student motivation, teacher immediacy and perceived sources of motivation and demotivation in college classes. Communication Education, 44, 292 306.

Decy, E.; \& Richard, R. (1985). Intrinsic motivation and self-determination in human behavior. Nueva York: Plenum. 
Department of Education and Skills (2017). Languages connect Ireland's strategy for foreign languages in education 2017-2026. Dublin: Department of Education and Skills. <https://bit.ly/2iNt7tK>

Department of Education of the Republic of Philippines (2012). Special Program in Foreign Language (SPFL). <https://bit.ly/2GfKMWe>

Dörnyei, Z. (1994). Motivation and motivating in the Foreign Language Classroom. The Modern Language Journal, 78 (iii), 273-284.

- (2001). Teaching and Researching Motivation. Nueva York: Pearson Education.

. (2009). The L2 motivational selfsystem. In Z. Dörnyei, \& E. Ushioda (Eds.), Motivation, Language Identity and the L2 Self. Clevedon: Multilingual Matters.

Dörnyei, Z.; \& Ryan, S. (2015). The Psychology of the Language Learner Revisited. Nueva York: Routledge.

Dörnyei, Z.; MacIntyre, P.; \& Alastair, H. (2015). Applying complex dynamic systems principles to empirical research on L2 motivation. In Z. Dörnyei, P. MacIntyre, \& H. Alastair (Eds.), Motivational Dynamics in Language Learning (pp. 1-10). Bristol: Multilingual Matters.

Erdoğan, E.; \& Tunaz, M. (2012). Determining External and Internal Demotivating Factors among Young Learners at Pozantı Regional Primary Boarding School. Frontiers of Language and Teaching, 3, 147-160. $<$ https://bit.ly/2rAfzTv>

Falout, J.; Elwood, J.; \& Hood, M. (2009). Demotivation: affective states and learning outcomes. System, 37, 403-417.

Falout, J.; \& Maruyama, M. (2004). A comparative study of proficiency and learner demotivation. The language teacher, 28 (8), 3-9.

Falout, J.; \& Falout, M. (2005). The other side of motivation: Learner demotivation. In K. Bradford-Watts, C. Ikeguchi, \& M. Swanson (Eds.), JALT2004. Conference proceedings. Tokio: JALT.

Falout, J. (2012). Coping with demotivation: EFL learners' remotivation processes. The Electronic Journal for Teaching English as a Foreign Language, 13 (3), 1-29.

Fauzi, N. (2014). Demotivation in learning English among pre-university students in the ESL classroom. Trabajo de fin de máster, Universidad Teknologi MARA, Malasia. <https://bit.ly/2BYvHUB>

Fujikawa, M. (2013). The most influential factors affecting the motivation and demotivation of Japanese university students studying English. Studies in British \& American literature, 48, 65-32.

Gardner, R.; \& Lambert, W.E. (1972). Attitudes and motivation in Second Language Learning. Rowley: Newbury House.

Gardner, R. (1985). Social psychology and second language learning: the role of attitude and motivation. London: Edward Arnold.

Gentry, M.; Gable, R. K.; \& Rizza, M. G. (2002). Students perceptions of classroom activities: are there grade-level and gender differences. Journal of Educational Psychology, 94, 539-544.

Ghadirzadeh, R.; Pourabolfathe Hashtroudi, F.; \& Shokri, O. (2012). Demotivating Factors for English Language Learning Among University Students. Journal of Social Sciences, 8 (2), 189-195. <https://bit.ly/2rqYHhG>

Ghonsooly, B.; Hassazandeh, T.; Samavarchi, L.; \& Hamedi, S. (2017). A mixed-methods approach to demotivating factors among Iranian EFL learners. Issues in Educational Research, 27(3), 417-434. <https://bit. ly/2RKppxc>

Gottlieb, N. (2011). Japan: Language Policy and Planning in Transition. Current Issues in Language Planning, $9(1), 1-68$. 
Gulnaz, F.; Ahmad, A.; \& Mandouh, S. Y. (2015). An exploration of the demotivational factors affecting teaching and learning of English as a foreign language of EFL learners in Gulf countries. International Journal of Social Sciences People, 2 (1), 17-32. <https://bit.ly/2Synjkf>

Hamada, Y.; \& Grafström, B. (2014). Demotivating factors in learning Japanese as a foreign language. 秋田 大学教育基礎教育研究年報, 9-14.<https://bit.ly/2Utm8Ez>

Hamada, Y.; \& Kito, K. (2008). Demotivation in Japanese high schools. In K. Bradford Watts, T. Muller, \& M. Swanson (Eds.), JALT2007 Conference Proceedings. Tokyo: JALT, 168-178. <https://bit.ly/2L4ueyX>

Hasegawa, A. (2004). Student demotivation in the Foreign Language Classroom. Takushoku Language Studies, 107, 119-136.

Heidari Soureshjani, K.; \& Riahipour, P. (2012). Demotivating Factors on English Speaking Skill: A Study of EFL Language Learners and Teachers' Attitudes. World Applied Sciences Journal, 17 (3), 327-339. <https:// bit.ly/2QisLuO>

Hirvonen, M. (2010). Demotivation in learning English among immigrant pupils in the ninth grade of comprehensive school. Trabajo de fin de grado, University of Jyväskylä, Finlandia. <https://bit.ly/2zPtqcP>

Hiver, P. (2015). Attractor states. In Z. Dörnyei, P. MacIntyre, \& H. Alastair (Eds.), Motivational Dynamics in Language Learning. Bristol: Multilingual Matters.

Hosseini, S. A.; \& Jafari, S. M. (2014). Possible demotivating factors for secondary school students. International Journal of Language Learning and Applied Linguistics World, 5(3), 188-201. <https://bit.ly/2QJ4QUG>

$\mathrm{Hu}, \mathrm{R}$. J. S. (2011). The relationship between demotivation and EFL Learners' English Language Proficiency. English Language Teaching, 4 (4), 88-96.

$\mathrm{Hu}$, Y. (2007). China's foreign language policy on primary English education: What's behind it? Language Policy, 6 (3-4), 359-376.

Hunealt, C. (2009). Estrategias motivacionales en el aula de ELE. Tinkuy, 11, 145-165. <https://bit. ly/2SyPEXx>

Instituto Cervantes (2018). El español, una lengua viva. Informe 2018. <https://cvc.cervantes.es/lengua/espanol_lengua_viva/>

Janedizadeh, S.; Ghanizadeh, A.; \& Ghonsooly, B. (2016). The role of EFL learners' demotivation, perceptions of classroom activities, and mastery goal in predicting their language achievement and burnout. Asian-Pacific Journal of Second and Foreign, 1:16. <DOI 10.1186/s40862-016-0021-8>

Jung, S. K. (2011). Demotivating and Remotivating Factors in Learning English: A Case of Low-Level College Students. English Teaching, 66 (2), 47-72.

Kaivanpanah, S.; \& Ghasemi, Z. (2011). An investigation into Sources of demotivation in second language learning. Iranian Journal of Applied Linguistics, 14 (2), 89-110.

Kaplan, R. B.; Baldauf Jr.; Richard B.; \& Kamwangamalu, N. (2011). Why educational language plans sometimes fail. Current issues in Language planning, 12 (2), 105-124.

Keblawi, F. (2005). Demotivation among Arab learners of English as a foreign language. In M. Singhal, \& J. Liontas (Eds.), Proceedings of the second international online conference on second and foreign language teaching and research. The reading matrix. United States. <https://bit.ly/2SyYG6Y>

Kikuchi, K.; \& Sakai, H. (2009). Japanese Learner's demotivation to Study English: a Survey study. JALT Journal, 31 (2), 183-203. <https://bit.ly/2G5oeHt>

Kikuchi, K. (2015). Demotivation in Second Language Acquisition. Insights from Japan. Bristol: Multilingual Matters. 
Kim, T. Y.; \& Seo, H. (2012). Elementary School Students Foreign Language Learning Demotivation A Mixed Methods Study of Korean EFL Context. The Asia-Pacific Education Researcher, 21 (1), 160-171. Disponible en web: https://bit.ly/2B4WeOe

Kim, Y. K.; \& Kim, T.Y. (2013). English learning demotivation studies in the EFL contexts: State of the Art. Modern English Education, 14 (1), 77-101.

Kim, S. (2015). Demotivation and L2 motivational self of Korean college students. English teaching, 70, 1, 29-55.

Kim, T. Y.; \& Kim, Y. K. (2015). Elderly Korean Learners' participation in English Learning through lifelong education: Focusing on motivation and demotivation. Educational Gerontology, 41, 120-135.

Kim, T. Y.; \& Kim, Y. (2016). EFL Learning demotivation in Korean Context: similarities and differences across schools' levels. English language \& Literature Teaching, 22 (1), 135-156.

Kim, T. Y.; Kim, Y.; \& Kim, J. Y. (2017). Structural relationship between L2 learning (de)motivation, resilience, and L2 proficiency among Korean College students. The Asia-Pacific Education Researcher, 26 (6), 397-406.

Krishnan, S. D.; \& Pathan, H. (2013). Investigating Demotivation in Learning English: An Extension to Sakai and Kikuchi's (2009) Framework. International Journal of English Linguistics, 7 (2), 124-131.<Doi:10.7575/ aiac.alls.v.4n.2p.124>

Li, L.; \& Zhou, C. (2013). Different faces of Demotivation: A comparative study on Chinese and Korean college EFL Learners' demotivators. Journal of Applied Sciences, 13, 800-809. <DOI: 10.3923/jas.2013.800.809.>

Macasová, H. (2014). Demotivation among English Language Students and How to Face it. Trabajo de fin de grado, Charles University in Prague, República Checa. <https://bit.ly/2L2JT1G>

Mahen, A.; \& Pathan, Z. (2017). Exploring Factors Causing Demotivation and Motivation in Learning English Language among College Students of Quetta, Pakistan. International Journal of English Linguistics, 7 (2), 81-89. < doi:10.5539/ijel.v7n2p81>

Marins de Andrade, P. R. (2010). Estrategias de aprendizaje y desarrollo de la motivación: un estudio empírico con estudiantes de E/LE brasileños. Porta Linguarum, 14, 141-160. <https://bit.ly/2Us8PnF>

Marins de Andrade, P. R.; \& Guijarro Ojeda, J. R. (2010). Afectividad y competencia existencial en estudiantes de español como lengua extranjera en Brasil. Revista de Lingüística Teórica y Aplicada, 48 (1), 51-74.

McClelland, N. (2000). Goal Orientations. In Japanese College Students Learning EFL. In S. Cornwell, \& P. Robinson (Eds.), Individual differences in foreign language learning: Effects of aptitude, intelligence and motivation. Tokio: Aoyama Gakuin University Press.

Méndez Santos, M. C. (2018a). Gestión de dinámicas de grupos en la enseñanza de ELE a adultos en contextos de instrucción formal: una pieza más del complejo fenómeno del aprendizaje. Foro de Profesores de ELE, 14, 175-183.

(2018b). Gestión de dinámicas de grupos en la enseñanza de ELE a adultos en contextos de instrucción formal: una pieza más del complejo fenómeno del aprendizaje. Revista Internacional de Lenguas Extranjeras, 9, 31-54.

Mendoza Puertas, J. D. (2017). Estudiantes coreanos de español y renuencia a hablar en clase. ¿Realidad o mito? Porta Linguarum, 28, 152-170.

Meshkat, M.; \& Hassani, M. (2012). Demotivating factors in learning English: the case of Iran”. Procedia Social and Behavioural Sciences, 3, 745-749. <https://bit.ly/2BYtF6V>

Midgley, C.; Kaplan, A.; Middleton, M.; Maehr, M. L.; Urdan, T.; Anderman, L. H.; Anderman, E.; \& Roeser, R. (1998). The development and validation of scales assessing students' achievement goal orientations. Contemporary Educational Psychology, 23(2), 113-31. 
Muhonen, J. (2004). Second language demotivation: Factors that discourage pupils from learning the English language. Trabajo de fin de grado, University of Jyvaskyla, Finlandia. <https://bit.ly/2L54zpU>

Muñoz Sanz, C. (2015). El alumno japonés en el aula de ELE: cómo lograr una comunicación eficaz. In Y. Morimoto, M. V. Pavón Lucero, \& R. Santamaría Martínez, (eds.), La enseñanza de ELE centrada en el alumno (pp. 681-690). Madrid: ASELE. <https://bit.ly/2E7FyZO>

Nguyen, H. B.; \& Thi Pham, V. T. (2017). English-major students' perceptions of demotivating factors in learning reading. International Journal of advanced research, 5 (8), 200-207. <https://bit.ly/2BX75LS>

Ohata, K. (2018). The Impact of Learner Demotivation: Retrospective Accounts of Japanese EFL Learners. Departmental Bulletin Paper. 1-21. <https://bit.ly/2rpzdBn>

Parente, M. C.; \& Pereira da Silva Schneider, M. S. (2013). A motivação e/ou a desmotivação na aula de língua inglesa dos alunos do $9^{\circ}$ ano: um estudo de caso. Anais do SILEL, 3 (1), 1-13. <https://bit.ly/2rrAA2v>

Quadir, M. (2017). Let us Listen to Our Students: An analysis of Demotivation to Study English in Bangladesh. The English teacher, 46 (3), 128-141. <https://bit.ly/2B3mKI4>

Rajavi, B.; \& Hosseini Pozveh, S.M. (2016). A Quantitative Analysis of Demotivating Factors for Iranian Intermediate High School EFL Learners. Mediterranean Journal of Social Sciences, 7 (5), 124-131. $<$ Doi:10.5901/mjss.2016.v7n5p124>

Rastegar, M.; Akbarzadeh, M.; \& Heidari, N. (2012). The darker side of motivation: Demotivation and its relation with two variables of Anxiety among Iranian Learners. International Scholarly Research Network, ISRN Education, 1-8.

Ryan, S. (2013). Motivation. In M. Byram, \& A. Hu (Eds.), Routdlege Encyclopedia of Language Teaching and Learning. Londres: Routledge.

Schaufeli, W. B.; Martínez, I.; Pinto, A. M.; Salanova, M.; \& Bakker, A. B. (2002). Burnout and engagement in university students: A cross-national study. Journal of Cross-Cultural Psychology, 33(5), 464-481.

Sharififar, M.; \& Akbarzadeh, M. (2011). An analysis of Demotivators in English classes for Iranian university students. Iranian EFL Journal, 7 (2), 28-40.

Shu-Chen, H. (2012). Pushing learners to work through tests and marks: Motivating or demotivating? A case in a Taiwanese University. Language Assessment Quaterly, 9 (1), 60-77.

Song, B.; \& Kim, T. Y. (2017). The dynamics of demotivation and remotivation among Korean high school EFL students. System, 65, 90-103.

Tabatabaei, O.; \& Molavi, A. (2012). Demotivating Factors Affecting EFL Learning of Iranian Seminary Students. International Education Studies, 5 (1), 181-190. <https://bit.ly/2UqrWOV>

Trang, T. T. T.; \& Baldauf, R. Jr. (2007). Demotivation: Understanding Resistance to English Language Learning - The Case of Vietnamese Students. The Journal of Asia TEFL, 4 (1), 79-105.

Tsuchiya, M. (2004a). Nihonjin daigakuseino eigogakushuuheno demotivation (Japanese university students' demotivation to study English). The Chugoku Academic Society of English Language Education Kenkyukiyo, 34, 57-66.

. (2004b). Factors in demotivation concerning learning English: A preliminary study of Japanese university students. The Kyushu Academic Society of English Language Education (KASELE), 32, 39-46.

. (2006a). Factors in demotivation of lower proficiency English learners at college. The Kyushu Academic Society of English Language Education (KASELE), 34, 87-96.

- (2006b). Profiling of lower achievement English learners at college in terms of demotivating factors. Annual Review of English Language Education in Japan (ARELE), 17, 171-180. 
Vallerand, R.; Briere, N.; Pelletier, L. \& Senecal, C. (2012). The academic motivation scale: A measure of intrinsic, extrinsic and amotivation in education. Educational and psychological measurement, 52, $1003-$ 1017.

Warrington, S. (2005). The Passivity / Demotivation Inventory, Center for English language education: Asia University.

Warrington, S.; \& Jeffrey, D. (2015). A Rationale for Passivity and De-Motivation Revealed: An Interpretation of Inventory Results Among Freshman English Students. Journal of Language and Learning, 3 (2), 312-335. <https://bit.ly/2QF59jq>

Weiner, B. (1992). Human motivation: Metaphors, Theories and Research. Newbury Park: Sage.

Williams, M.; \& Burden, R. (1997). Psychology for Language teachers. Cambridge: Cambridge University Press.

Xuezheng, J. (2014). Investigation of Learner Demotivation in English Learning in Chinese College. International Journal on Studies in English Language and Literature (IJSELL), 2 (12), 39-45. <https://bit.ly/2EmokbQ>

Zach, C. (2016). The Role of (De)motivational Factors in the Context of Learning. English in South Korea. Tesis doctoral no publicada. Stellenbosch University, Sudáfrica. <https://scholar.sun.ac.za> 